\title{
Study of Correlation of Retinal Layer Thickness with Duration and Severity of Parkinson's Disease
}

\author{
Authors \\ Dr Kunal B Jadhav ${ }^{1}$, Dr R Balakrishnan ${ }^{2}$, Madhavi Karri ${ }^{3}$ \\ ${ }^{1}$ Final year D.M Neurology Student, ${ }^{2}$ Prof. and H.O.D Dept of Neurology, ${ }^{3}$ 1st Year D.M. Neurology \\ Student \\ Corresponding Author \\ Dr Kunal B Jadhav \\ Neurology Dept PSG Hospital IMS\&R, Coimbatore, Tamil Nadu, India \\ Email: kunal110284@gmail.com
}

\begin{abstract}
$P D$ is primarily a motor disorder associated with loss of dopaminergic neurons. Dopamine (DA) is also a major neurotransmitter in the retina. There is concurrent retinal DA neurons loss in in the inner nuclear \&inner plexiform layers in PD. OCT is analogous to ultrasound \& its provides detailed cross sectional images of retina.

Aim: To do the macular scans, parapapillary RNFL in vivo \& to evaluate whether retinal structural changes are seen in $P D$ \&its relation to the clinical severity of the $P D$.

Materials \& Method: Our prospective study was conducted in PSG Hospital IMS\&R. Spectral OCT Was used to examined the RNFL thickness in all quadrants \& Macular volume in PD patients without visual impairment $(n=25)$ \& compared them with healthy age, sex matched control $(n=25)$. PD severity will be assessed using the Hoehn \&Yahr scale.

Results: Compared to healthy control the thickness in the inferior quadrant of PD patients (149 \pm 6 microns)

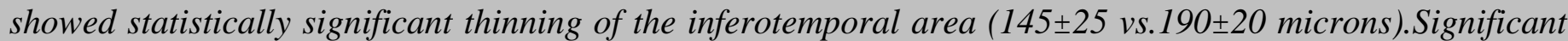
differences were observed between controls and patients in relation to mean macular thickness PD duration \& severity were inversely correlated

Conclusion: RNFL thickness \& macular thickness could be a surrogate biomarker for PD diagnosis in early stage aid in monitoring treatment effectiveness \& monitoring disease progression in PD.

Keywords- Parkinson's disease Retina; Retinal nerve fiber layer; Optical coherence tomography.
\end{abstract}

\section{Introduction}

Parkinson's disease (PD) is primarily a motor disorder associated with progressive degeneration of dopaminergic neurons in the substantia nigra pars compacts characterised by presence of $\alpha$-synuclein Lewy bodies ${ }^{[1]}$ As detailed picture of PD is emerging as a result of extensive research being done all over the world, the non motor manifestations of PD being the main focus over the last few years. Most prominent non motor symptoms in PD are sleep disorders, cognitive and behavioural changes, autonomic failure and visual symptoms ${ }^{[2]}$ As the PD advances the there is loss of dopaminergic neurons in substantia niagra pars compacta and Neurons or neuronal circuits outside the brain can be affected simultaneously or even before the substantia nigra. ${ }^{[3]}$ 
Visual System and Dopamine: The human retina ganglion cells main role in color vision and contrast Sensitivity. The retina has dopaminergic neurons within the inner retinal layer. Dopamine helps in modulating these ganglion cells through lateral interactions especially in inner plexiform layer. Hence Dopamine has crucial role vision. ${ }^{[4]}$

\section{Retinal Involvement in Parkinson Disease}

Patients with PD commonly complain of impaired visual function and difficulty reading ${ }^{[6]}$. Dopamine is required for absolute sensitivity, spatial contrast sensitivity, temporal sensitivity and colour vision impaired in $\mathrm{PD}{ }^{[4,6,7]}$.

Dopamine deficiency and dopaminergic neuronal degeneration in retina leading to abnormal perception of colour and motion reduced contrast sensitivity in $\mathrm{PD}^{[8,9,10,11,12]}$

Impaired vision in PD was first shown by psychophysical and visual evoked potential (VEP) measurements [13,14]

Post-Mortem Studies: The DA content of the retina as measured at post-mortem, is low in PD. Dopaminergic neuronal cells have been identified in the inner nuclear and inner plexiform layers, there is concurrent retinal dopamine neurons loss in PD [15]

PD and the role of retina as a model of neurodegeneration

Thinning of the retinal nerve fibre layer (RNFL), the inner retinal layer (IRL), and macular thickness have been Documented in few studies, and it has been proposed that this may correlate with loss of these dopaminergic cells and progression of functional visual abnormalities in PD patients ${ }^{[16,17]}$

\section{OCT scan}

OCT is a new non-invasive, imaging technology which provides very high resolution, cross sectional images retina and measure anatomic layers of the retina.[Fig 1]

The principle of OCT is analogous to ultrasound; however, the system uses light instead of acoustic waves. OCT scans takes circular scan around the
ONH provide reliable measurements of the retinal nerve fiber layer thickness (RNFL) ${ }^{[18]}$

\section{FIG 1}

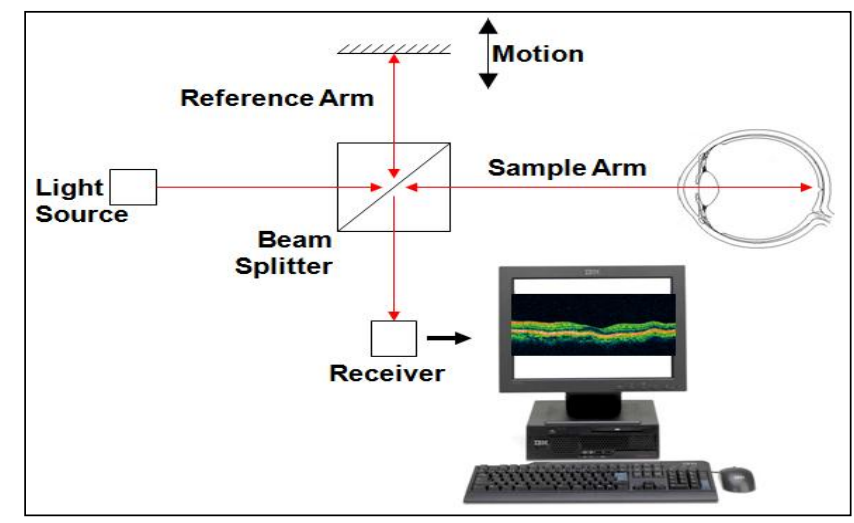

At present, the development of therapy in PD is hampered by the lack of well-established biomarkers that can assist with diagnosis and/or tracking the progression of the disease. Aim of this study is to OCT in PD patient and measure the changes of retinal nerve fibre and macular volume and to evaluate whether these changes in RNFL and macular volume can be used as a marker for the disease course by PD.

\section{Aim}

1. The aim of this study is to assess the peripapillary retinal nerve fibre layer (RNFL) and macular thickness in patients with Parkinson's disease (PD).

2. To assess the Correlation of Retinal Layer Thickness and macular thickness with Duration and Severity of Parkinson's Disease

\section{Study Design}

This was an observational, prospective crosssectional study

\section{Material and Methods}

PD patients came to neurology Clinic were recruited for the study.

The criteria for PD diagnosis according to Brain Bank criteria

1. at least two of the following signs: rest tremor, rigidity, bradykinesia, and postural reflex impairment, 
2. Known sustained response to antiparkinsonian medication

3. no other known or suspected causes for parkinsonism.

\section{Exclusion Criteria}

1.Patients with posterior pole pathology such as macular degeneration, those with optic neuropathies including glaucoma, glaucoma suspect or patients with media pacification such as cataract that precluded precise ocular and OCT examinations were excluded.

2. DM. Patients

\section{Procedure}

The Institutional Ethics Committee approved the study.

All subjects signed an informed consent for participation.

Each patient underwent ocular examination, intraocular pressure measurement and visual field (VF) examination

Each eye was examined by OCT (second generation Fourier-domain device), with the pupil dilated.

The subject was asked to fixate a luminous point; the required area was scanned by OCT.

The examination included five circumpapillary standard circular scans $(3.4 \mathrm{~mm}$ in diameter $)$ followed by six radial lines centered at the optic nerve head (6 mm length each).

Macular volume and thickness scan was done for all patients.

\section{Data}

30 PD patients were initially recruited. Five were excluded: four were unable to complete the OCT examination (dyskinesia, tremor, fatigue), one due to newly diagnosed glaucoma 25 PD OCT scans of PD patients and 25 age-matched controls were also examined followed y OCT scan for RNFL , Macular volume. Parkinson's disease severity was assessed using the Hoehn and Yahr Scale

\section{Statistical Analysis}

All data analyses were performed using statistical software (SPSS version 15; IBM Corporation New York). Parameters between PD patients and healthy were compared by Student's t-test. P- value of less than 0.05 were considered statistical significance. Pearson correlation coefficient was used to evaluate linear correlation between RNFL structural, macular volume and Hoehn and Yahr scales. The RNFL thickness was measured in Superior, Inferior, Nasal, Temporal, Temporal \& Superior, Nasal Superior, Nasal Inferior, Temporal \& Inferior quadrants, in 25 PD patients and was compared to age- sex matched control using independent t-test. Macular volume and thickness was measured in four retinal quadrants, namely: superior, inferior, nasal and temporal quadrants in PD patients and was compared with that of age and sex -matched control subjects by independent t-test..

\section{Results}

1. Epidemiologic in Parkinson's disease cohort showed 16 were male and 9 were female patients with mean age of 62 years. Hoehn and Yahr score showed fifteen patients were in stage 1 , two in stage 2 and eight patient in stage 3 , Mean duration of PD was 6 years among patients

2. In PD patients RNFL thickness in the inferior retinal quadrant (149 \pm 6 microns) was significantly thinner than in control subjects $(183 \pm 21$ microns; $\mathrm{p}=0.001)$

3. The RNFL thickness at the inferotemporal site in PD patients ranged between 104 and 233 microns

4. Within the inferior quadrant, the RNFL was the thinnest in the inferotemporal area (146 \pm 23 microns) as compared with controls (187 \pm 20 microns; $\mathrm{p}=0.001$ ).

5. Individual values RNFL thickness inferior temporal quadrant of control subjects were: 164, 167, 172,180, 192, 196, 199, 204, 199, $232,163,163,170,179,190,195,198,190$, 104, 233 162, 168, 171,182, 190, microns. 
6. The inferotemporal thickness was $>200$ microns in twenty control subjects and in only three of the $25 \mathrm{PD}$

7. The results show significant loss of RNFL thickness in PD at specific sites.

8. no statistical correlation was found between parkinson disease duration or severity staging with RNFL thickness in OCT scan

9. avarage Macular volume was positively correlating correlated with disease duration and Hoehn and Yahr scale

\section{Table 1}

\begin{tabular}{|l|c|c|}
\hline H \& Y STAGE of 25 PD patient \\
\hline H\&Y Stage & Total & Percent \\
\hline 1 & 15 & $60 \%$ \\
\hline 2 & 2 & $8 \%$ \\
\hline 3 & 8 & $32 \%$ \\
\hline
\end{tabular}

Table 2

Relation between PD Duration and Macular Volume

\begin{tabular}{|l|c|c|c|c|}
\hline & PD Duration & No. & Mean & P VALUE \\
\hline RT MACULAR & $<6 \mathrm{yrs}$ & 15 & 8.0880 & 0.001 \\
VOLUME & $<6 \mathrm{yrs}$ & 9 & 5.4567 & \\
\hline
\end{tabular}

Table 3

\begin{tabular}{|c|c|c|c|c|}
\hline \multicolumn{5}{|c|}{ Comparing Average Macular Volume in PD and control } \\
\hline & group & $\mathrm{No}$ & Mean & P VALUE \\
\hline RT MACULAR VOLUME & $\begin{array}{c}\text { PD } \\
C\end{array}$ & \begin{tabular}{l|}
25 \\
25
\end{tabular} & $\begin{array}{l}6.9560 \\
7.7792\end{array}$ & 0.027 \\
\hline
\end{tabular}

\section{Table 4}

\begin{tabular}{|c|c|c|c|}
\hline \multicolumn{4}{|c|}{ Comparison between RNFL in PD and control } \\
\hline RNFL in different quadrants & No & Mean & P VALUE \\
\hline \multirow{2}{*}{ RT RNFL TS } & 25 & 120.76 & \multirow{10}{*}{$>0.05$} \\
\hline & 25 & 182.63 & \\
\hline \multirow[t]{2}{*}{ RT RNFL NS } & 25 & 118.72 & \\
\hline & 25 & 174.08 & \\
\hline \multirow[t]{2}{*}{ RT RNFL S } & 25 & 123.0936 & \\
\hline & 25 & 173.9167 & \\
\hline \multirow[t]{2}{*}{ RT RNFL N } & 25 & 93.00 & \\
\hline & 25 & 127.71 & \\
\hline \multirow[t]{2}{*}{ RT RNFL NI } & 25 & 112.40 & \\
\hline & 25 & 155.25 & \\
\hline \multirow[t]{2}{*}{ RT RNFL TI } & 25 & 102.44 & \multirow[t]{4}{*}{$<0.05$} \\
\hline & 25 & 182.25 & \\
\hline \multirow[t]{2}{*}{ RT TNFL I } & 25 & 124.5140 & \\
\hline & 25 & 167.4167 & \\
\hline \multirow[t]{2}{*}{ RT RNFL T } & 25 & 77.52 & $>0.05$ \\
\hline & 25 & 135.63 & \\
\hline \multirow{2}{*}{$\begin{array}{ll}\text { RNFL thickness } & \text { PI } \\
& \mathrm{C} \\
\end{array}$} & 25 & 105.064000 & $<0.05$ \\
\hline & 25 & 156.729167 & \\
\hline \multicolumn{4}{|c|}{$\begin{array}{l}\text { RNFL- Retinal Nerve Fibre Layer, TS -Temporal \& Superior, NS- Nasal } \\
\text { Superior, S -Superior, N -Nasal, NI-Nasal Inferior, TI- Temporal \& } \\
\text { Inferior, I- Inferior, T- Temporal }\end{array}$} \\
\hline
\end{tabular}

\section{Discussion}

We selected patients in all stages of PD to confirm the hypothesis whether RNFL thickness correlating with progression of PD.

Our study show significant reductions in RNFL thickness between idiopathic PD patients and agematched controls. and this difference was most prominent in the inferotemporal quadrant

This findings are in agreement with the findings of Altinas et al, which showed thinning macula .8 [Altintas O, Iseri P, Ozkan B, Caglar Y. Correlation between retinal morphological and functional findings and clinical severity in Parkinson's disease. Doc Ophthalmol. 2008;116:137-146. [PubMed]

Some studies like Inzelberg et al ${ }^{(19)}$ and Moschos et al ${ }^{(20)}$ found selective thinning of RNFL in the temporal quadrant, whereas other reports documented thinning in the inferior ${ }^{(20,19)}$, and nasal ${ }^{(21)}$ quadrants.

Our study also show significant correlation in average macular thickness with disease duration and Hoehn and Yahr scale in PD patients as compaired to and age-matched controls. This was not shown in studies till now

These structural changes in retina in PD patients is due to dopaminergic neuronal loss in the retina, this dopaminergic neuronal loss have downstream effects ${ }^{[22]}$ which affects the visual processing by altering of receptive field properties of ganglion cells, ${ }^{[23]}$ whose axons form the Retinal nerve layers . Dopamine has neurotropic effect in retina maintains the structural integrity of retina.

\section{Conclusions}

With our study results and previous study reports we postulate that RNFL thinning as result of DA depletion is hallmark of retinal changes in PD. OCT is a valuable tool in evaluating PD. OCT may provide a valid and reliable biomarker to tracing neurodegeneration within the retina and a primary outcome measure to detect the effects of new therapeutic strategies and follow-up of disease progression of PD. Also, this biomarker may be useful in identifying the disease early in the course so that early treatment can be started 


\section{Limitations}

Overall, ours is small,. However, our sample size of twenty five patients does not differ greatly from previously published studies findings (by Hajee et al Inzelberg et al, Altinas et al.) Future study with larger cohort of PD patients, followed prospectively for disease progress with multiple OCT scan is needed to clarify the precise relationship between retinal changes and $\mathrm{PD}$.

\section{References}

1. Spillantini MG Nature. 1997;388 (6645):839-840

2. Park A, Stacy M. Non-motor symptoms in Parkinson's disease. J Neurol 2009; 256(Suppl 3): 293-298

3. Braak H, Del Tredici K, Bratzke H, HammClement J, Sandmann-Keil D, Ru“b U. Staging of the intracerebral inclusion body pathology associated with idiopathic Parkinson's disease (preclinical and clinical stages). J Neurol 2002; 249(Suppl 3): III/1III/5

4. (Djamgoz, Hankins, Hirano, \& Archer, 1997).

5. Harnois C, Di Paolo T. Decreased dopamine in the retinas of patients with Parkinson's disease. Invest Ophthalmol Vis Sci. 1990;31(11)

6. Bodis-Wollner I. Visual deficits related to dopamine deficiency in experimental animals and Parkinson's disease patients. Trends Neurosci. 1990;13(7):

7. Jackson GR, Owsley C. Visual dysfunction, neurodegenerative diseases, and aging. Neurol Clin. 2003;21(3)

8. Matsui H, Udaka F, Tamura A, Oda M, Kubori T, Nishinaka K, et al. Impaired

9. Bodis-Wollner I, Marx M, Mitra S, Bobak P, Mylin L, Yahr M. Visual dysfunction in Parkinson's disease. Brain 1987;110:1675e98.

10. Price MJ, Feldman RG, Adelberg D, Kayne $\mathrm{H}$. Abnormalities in color vision and contrast sensitivity in Parkinson's disease. Neurology 1992;42:887e90.

11. Pieri V, Diederich NJ, Raman R, Goetz CG. Decreased colour discrimination and contrast sensitivity in Parkinson's disease. Journal of the Neurological Sciences 2000;172:7e11.

12. Uc EY, Rizzo M, Anderson SW, Qian S, Rodnitzky RL, Dawson JD. Visual dysfunction in Parkinson disease without dementia. Neurology 2005;65: 1907e13.

13. Dyer RS, Howell WE, MacPhail RC. Dopamine depletion slows retinal transmission. Exp Neurol. 1981;71(2)

14. Onofrj M, Bodis-Wollner I. Dopaminergic deficiency causes delayed visual evoked potentials in rats. Ann Neurol. 1982;11(5):

15. I. Di Paolo T. Harnois C. and Daiglc M: Assay of dopamine and its metabolites in human and rai retina. Ncurosci Leu 74:250, 1987.

16. Parkinson's disease patients. Eye. 2013;27:507-514.

17. Garcia-Martin E, Satue M, Fuertes I, et al. Ability and reproducibility of Fourierdomain optical coherence tomography to detect retinal nerve fiber layer atrophy in Parkinson's disease. Ophthalmology. 2012;119:2161-2167.

18. Blumenthal EZ, Weinreb RN. Assessment of the retinal nerve fiber layer in clinical trials of glaucoma neuroprotection. Survey Ophthalmol. 2001;45:305-312. discussion 332-334

19. Inzelberg R, Ramirez JA, Nisipeanu $P$, Ophir A. Retinal nerve fi ber layer thinning in arkinson disease. Vision Res. 2004;44:2793 - 279

20. Moschos MM, Tagaris G, Markopoulos I, Margetis I, Tsapakis S, Kanakis M, Koutsandrea C. Morphologic changes and functional retinal impairment in patients with Parkinson disease without visual loss. Eur J Ophthalmol. 011:21;24 - 29 
21. Altintas O, Iseri P, Ozkan B, Caglar Y. Correlation between retinal morphological and functional fi ndings and clinical severity in Parkinson' $\mathrm{s}$ disease. Doc Ophthalmol. 2008; 116:137 - 146.

22. Jackson, G. R., \& Owsley, C. (2003). Visual dysfunction, neurode- generative diseases and aging. Neurology Clinics of North America, 21, 709-728.).

23. Djamgoz MB, Hankins MW, Hirano J, Archer SN. Neurobiology of retina dopamine in relation to degenerative states of the tissue. Vision Res. 1997;37:35093529. [PubMed] 